\title{
Convex Cones, Lyapunov Functions, and the Stability of Switched Linear Systems
}

\author{
Robert Shorten, Oliver Mason, and Kai Wulff \\ Hamilton Institute, NUI Maynooth, Ireland \\ robert.shorten@may.ie
}

\begin{abstract}
Recent research on switched and hybrid systems has resulted in a renewed interest in determining conditions for the existence of a common quadratic Lyapunov function for a finite number of stable LTI systems. While efficient numerical solutions to this problem have existed for some time, compact analytical conditions for determining whether or not such a function exists for a finite number of systems have yet to be obtained. In this paper we present a geometric approach to this problem. By making a simplifying assumption we obtain a compact time-domain condition for the existence of such a function for a pair of LTI systems. We show a number of new and classical Lyapunov results can be obtained using our framework. In particular, we demonstrate that our results can be used to obtain compact time-domain versions of the SISO KalmanYacubovich-Popov lemma, the Circle Criterion, and stability multiplier criteria. Finally, we conclude by posing a number of open questions that arise as a result of our approach.
\end{abstract}

\section{Introductory Remarks}

The Kalman-Yacubovich-Popov lemma has played an important role in the development of adaptive control algorithms. The classical KYP lemma is closely related to the existence of a common quadratic Lyapunov function for Lur'e type systems and is typically expressed in the form of a constraint on the Nyquist curve of a transfer function. In this paper we present a result on the existence of a quadratic Lyapunov function for a class of time-varying systems [1. As well as leading to new results in stability theory, we also show that our result reveals an interesting connection between the time and frequency domain 2, 33 for SISO systems. We use this connection to derive a compact time-domain version of the KYP lemma for SISO systems. Further, we show that for SISO systems, timedomain versions of many multiplier criteria can be obtained directly from their frequency domain counterparts [4]. Finally, we present a number of interesting open questions that arise as a result of our work.

\section{Mathematical Preliminaries}

Throughout, the following notation is adopted: $\mathbb{R}$ and $\mathbb{C}$ denote the fields of real and complex numbers respectively; $\mathbb{R}^{n}$ denotes the $n$-dimensional real Euclidean 
space; $\mathbb{R}^{n \times n}$ denotes the space of $n \times n$ matrices with real entries; $x_{i}$ denotes the $i^{\text {th }}$ component of the vector $x$ in $\mathbb{R}^{n} ; a_{i j}$ denotes the entry in the $(i, j)$ position of the matrix $A$ in $\mathbb{R}^{n \times n}$.

The main results of this paper are based upon Theorem 1 The concepts of weak quadratic Lyapunov functions, strong quadratic Lyapunov functions, and matrix pencils, are central to the statement of this theorem.

(i) Strong and weak common quadratic Lyapunov functions : Consider the set of LTI systems

$$
\Sigma_{A_{i}}: \dot{x}=A_{i} x, i \in\{1,2, \ldots M\} .
$$

where $M$ is finite and the $A_{i}, i \in\{1,2, \ldots M\}$, are constant Hurwitz matrices in $\mathbb{R}^{n \times n}$ (i.e. the eigenvalues of $A_{i}$ lie in the open left half of the complex plane and hence the $\Sigma_{A_{i}}$ are stable LTI systems). Let the matrix $P=P^{T}>$ $0, \quad P \in \mathbb{R}^{n \times n}$, be a simultaneous solution to the Lyapunov equations

$$
A_{i}^{T} P+P A_{i}=-Q_{i}, i \in\{1,2, \ldots M\} .
$$

Then, $V(x)=x^{T} P x$ is a strong quadratic Lyapunov function for the LTI system $\Sigma_{A_{i}}$ if $Q_{i}>0$, and is said to be a strong CQLF for the set of LTI systems $\Sigma_{A_{i}}, i \in\{1, \ldots, M\}$, if $Q_{i}>0$ for all $i$. Similarly, $V(x)$ is a weak quadratic Lyapunov function for the LTI system $\Sigma_{A_{i}}$ if $Q_{i} \geq 0$, and is said to be a weak $C Q L F$ for the set of LTI systems $\Sigma_{A_{i}}, i \in\{1, \ldots, M\}$, if $Q_{i} \geq 0$ for all $i$.

(ii) The matrix pencil $\sigma_{\gamma[0, \infty)}\left[A_{1}, A_{2}\right]$ : The matrix pencil $\sigma_{\gamma[0, \infty)}\left[A_{1}, A_{2}\right]$, for $A_{1}, A_{2} \in \mathbb{R}^{n \times n}$, is the parameterised family of matrices $\sigma_{\gamma[0, \infty)}\left[A_{1}, A_{2}\right]=A_{1}+\gamma A_{2}, \gamma \in[0, \infty)$. We say that the pencil is non-singular if $\sigma_{\gamma[0, \infty)}\left[A_{1}, A_{2}\right]$ is non-singular for all $\gamma \geq 0$. Otherwise the pencil is said to be singular. Further, a pencil is said to be Hurwitz if its eigenvalues are in the open left half of the complex plane for all $\gamma \geq 0$. It is important for much of what follows to note that when $A_{1}$ is non-singular, the pencil $\sigma_{\gamma[0, \infty)}\left[A_{1}, A_{2}\right]$ is non-singular if and only if the product $A_{1}^{-1} A_{2}$ has no negative eigenvalues.

The relationship between a matrix, its inverse, and a quadratic Lyapunov function will arise in our discussion. In this context we note the following fundamental result.

(iii) The stability of $\Sigma_{A}$ and $\Sigma_{A^{-1}}$ [5, 6]:

Consider the linear time invariant systems

$$
\begin{aligned}
\Sigma_{A} & : \dot{x}=A x, \\
\Sigma_{A^{-1}} & : \dot{x}=A^{-1} x,
\end{aligned}
$$

where $A \in \mathbb{R}^{n \times n}$ is Hurwitz. Then, any quadratic Lyapunov function for $\Sigma_{A}$ is also a quadratic Lyapunov function for $\Sigma_{A^{-1}}$. 
Comment : Suppose that $V(x)$ is a CQLF for the two stable LTI systems $\Sigma_{A_{1}}, \Sigma_{A_{2}}$. It is a simple exercise in algebra to verify that the same function $V(x)$ will be a quadratic Lyapunov function for the systems $\Sigma_{\sigma_{\gamma[0, \infty)}\left[A_{1}, A_{2}\right]}$ and $\Sigma_{\sigma_{\gamma[0, \infty)}\left[A_{1}, A_{2}^{-1}\right]}$ for all $\gamma \in[0, \infty)$. Hence, $\sigma_{\gamma[0, \infty)}\left[A_{1}, A_{2}\right]$ and $\sigma_{\gamma[0, \infty)}\left[A_{1}, A_{2}^{-1}\right]$ are both necessarily Hurwitz for all $\gamma \in[0, \infty)$. Thus the non-singularity of these two pencils is a necessary condition for the existence of a CQLF for the systems $\Sigma_{A_{1}}, \Sigma_{A_{2}}$.

Finally, the following observations are useful in deriving the results in Section 5 Lemma 2.1 is a well known result from linear algebra and Lemma 2.2 is a generalisation of a result used by Kalman in [7].

(iv) Lemma 1. [8] Let $A \in \mathbb{R}^{n \times p}, B \in \mathbb{R}^{p \times n}$, and let $I_{n}$ denote the $n \times n$ identity matrix. Then,

$$
\operatorname{det}\left[I_{n}-A B\right]=\operatorname{det}\left[I_{p}-B A\right] .
$$

(v) Lemma 2. [7] Let $A \in \mathbb{R}^{n \times n}$, and $c, b \in \mathbb{R}^{n \times 1}$. Then, the numerator and denominator polynomials of the rational function,

$$
1+\operatorname{Re}\left\{c^{T}\left(j \omega I_{n}-A\right)^{-1} b\right\}=\frac{\Gamma\left(-\omega^{2}\right)}{|M(j \omega)|^{2}},
$$

are given by

$$
\begin{aligned}
|M(j \omega)|^{2} & =\operatorname{det}\left[\omega^{2} I_{n}+A^{2}\right], \\
\Gamma\left(-\omega^{2}\right) & =\left(1-c^{T} A\left(\omega^{2} I_{n}+A^{2}\right)^{-1} b\right) \operatorname{det}\left[\omega^{2} I_{n}+A^{2}\right] .
\end{aligned}
$$

When the matrix $A$ is Hurwitz, $\operatorname{det}\left[\omega^{2} I_{n}+A^{2}\right]=\operatorname{det}[A] \operatorname{det}\left[\omega^{2} A^{-1}+A\right] \neq$ $0 \forall \omega \in \mathbb{R}$.

\section{A Result on Common Quadratic Lyapunov Functions}

The following theorem considers pairs of stable LTI systems for which no strong CQLF exists, but for which a weak CQLF exists with $Q_{i}, i \in\{1,2\}$, of rank $n-1$ in (2), and establishes a set of easily verifiable algebraic conditions, that are satisfied when such a weak CQLF exist:11. It will be later shown that these conditions are found to play an important role in the question of the existence of strong CQLF's for general LTI systems.

Theorem 1. Let $A_{1}, A_{2}$ be two Hurwitz matrices in $\mathbb{R}^{n \times n}$ such that a solution $P=P^{T}>0$ exists to the non-strict Lyapunov Equations

$$
A_{1}^{T} P+P A_{1}=-Q_{1} \leq 0,
$$

\footnotetext{
${ }^{1}$ This situation corresponds to two stable LTI systems that are on the boundary of having a CQLF as depicted in Figure 1.
} 


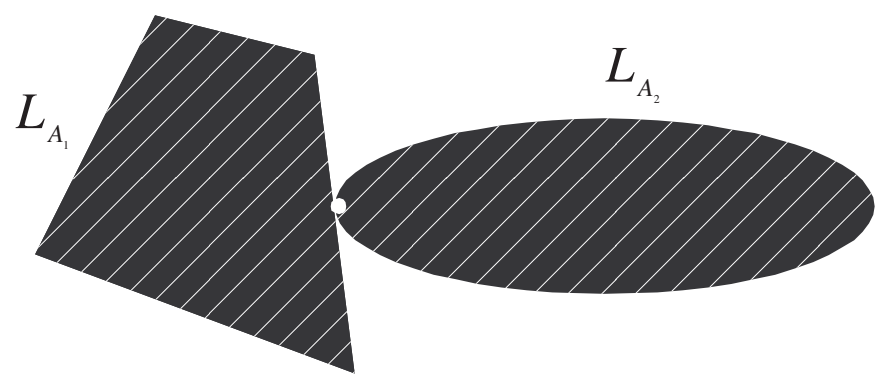

Fig. 1. The sets $L_{A_{i}}$ are defined by $\left\{P=P^{T}>0: A_{i}^{T} P+P A_{i} \leq 0\right\}$

$$
A_{2}^{T} P+P A_{2}=-Q_{2} \leq 0,
$$

for some positive semi-definite matrices $Q_{1}, Q_{2}$ both of rank $n-1$. Furthermore suppose that no solution exists to the strict Lyapunov equations (2). Under these conditions, at least one of the pencils $\sigma_{\gamma[0, \infty)}\left[A_{1}, A_{2}\right], \sigma_{\gamma[0, \infty)}\left[A_{1}, A_{2}^{-1}\right]$ is singular, and at least one of the matrix products $A_{1} A_{2}$ and $A_{1} A_{2}^{-1}$ has a real negative eigenvalue.

Outline of proof: As $Q_{1}$ and $Q_{2}$ are of rank $n-1$, there are non-zero vectors $x_{1}, x_{2}$ such that

$$
\begin{aligned}
& x_{1}^{T} Q_{1} x_{1}=0 \\
& x_{2}^{T} Q_{2} x_{2}=0 .
\end{aligned}
$$

The proof of Theorem 3.1 is split into two main stages.

Stage 1: The first stage in the proof is to show that if there exists a positive

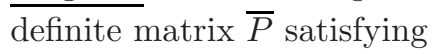

$$
\begin{aligned}
& x_{1}^{T} \bar{P} A_{1} x_{1}<0 \\
& x_{2}^{T} \bar{P} A_{2} x_{2}<0
\end{aligned}
$$

then a strong positive definite solution exists to (2).

Note that as $x^{T} P A_{1} x$ is a scalar for any $x$, we can write $x^{T} Q_{1} x=2 x^{T} P A_{1} x$. The same obviously holds for $x^{T} Q_{2} x$.

Now assume that there is some $\bar{P}$ satisfying (9), (10), and, firstly, consider the set

$$
\Omega_{1}=\left\{x \in \mathbb{R}^{n}:\|x\|=1 \text { and } x^{T} \bar{P} A_{1} x \geq 0\right\} .
$$

Here $\|x\|$ is the usual Euclidean norm on $\mathbb{R}^{n}$. The function that takes $x$ to $x^{T} \bar{P} A_{1} x$ is continuous. Thus $\Omega_{1}$ is closed and bounded, hence compact. Furthermore $x_{1}$ (or any non-zero multiple of $x_{1}$ ) is not in $\Omega_{1}$ and thus $x^{T} P A_{1} x$ is strictly negative on $\Omega_{1}$. 
Let $M_{1}$ be the maximum value of $x^{T} \bar{P} A_{1} x$ on $\Omega_{1}$, and let $M_{2}$ be the maximum value of $x^{T} P A_{1} x$ on $\Omega_{1}$. Then by the final remark in the previous paragraph, $M_{2}<0$. Choose any constant $\delta_{1}>0$ such that

$$
\delta_{1}<\frac{\left|M_{2}\right|}{M_{1}+1}
$$

and consider the positive definite matrix

$$
P+\delta_{1} \bar{P}
$$

By separately considering the cases $x \in \Omega_{1}$ and $x \notin \Omega_{1},\|x\|=1$, it is easy to see that for all non-zero vectors $x$ of norm 1

$$
x^{T}\left(A_{1}^{T}\left(P+\delta_{1} \bar{P}\right)+\left(P+\delta_{1} \bar{P}\right) A_{1}\right) x<0
$$

provided $0<\delta_{1}<\frac{\left|M_{2}\right|}{M_{1}+1}$. But we can scale $x$ by any real constant without changing this inequality. Thus $A_{1}^{T}\left(P+\delta_{1} \bar{P}\right)+\left(P+\delta_{1} \bar{P}\right) A_{1}$ is negative definite. Let $C_{1}$ denote the value $\frac{\left|M_{2}\right|}{M_{1}+1}$.

NOTE: It may appear that we are assuming that the set $\Omega_{1}$ is non-empty. However, if $\Omega_{1}$ was empty, then any positive constant $\delta_{1}$ could be used in the argument above to make $A_{1}^{T}\left(P+\delta_{1} \bar{P}\right)+\left(P+\delta_{1} \bar{P}\right) A_{1}$ negative definite.

Now the same argument can be used to guarantee the existence of a positive constant $C_{2}$ such that

$$
x^{T}\left(A_{2}^{T}\left(P+\delta_{1} \bar{P}\right)+\left(P+\delta_{1} \bar{P}\right) A_{2}\right) x<0 .
$$

for all non-zero $x$ provided we choose $0<\delta_{1}<C_{2}$. So, if we choose $\delta$ less than the minimum of $C_{1}, C_{2}$, we would have a positive definite matrix

$$
P_{1}=P+\delta \bar{P}
$$

which was a solution of (2).

Stage 2 : So under our assumptions, no positive definite solution $\bar{P}$ exists satisfying Equations (9) and (10). We now show that such a solution $\bar{P}$ would exist unless one of the two pencils $\sigma_{\gamma[0, \infty)}\left[A_{1}, A_{2}\right], \sigma_{\gamma[0, \infty)}\left[A_{1}, A_{2}^{-1}\right]$ was singular.

Recall (17), (8)) that there is a positive definite $P$ such that

$$
\begin{aligned}
& x_{1}^{T} P A_{1} x_{1}=0 \\
& x_{2}^{T} P A_{2} x_{2}=0 .
\end{aligned}
$$

Suppose now that there was a Hermitian matrix $H$ such that

$$
\begin{gathered}
x_{1}^{T} H A_{1} x_{1}<0 \\
x_{2}^{T} H A_{2} x_{2}<0 .
\end{gathered}
$$


As the set of positive definite matrices is open in the set of Hermitian matrices, we could choose $\epsilon>0$ such that $P+\epsilon H$ was positive definite. Then $P+\epsilon H$ would satisfy (9), (10). So in fact, there is no Hermitian $H$ satisfying (13), (14). This means that any Hermitian $H$ that makes the expression $x_{1}^{T} H A_{1} x_{1}$ negative will make the expression $x_{2}^{T} \mathrm{H}_{2} x_{2}$ positive.

More formally

$$
x_{1}^{T} H A_{1} x_{1}<0 \Longleftrightarrow x_{2}^{T} H A_{2} x_{2}>0
$$

for Hermitian $H$. It follows from this that

$$
x_{1}^{T} H A_{1} x_{1}=0 \Longleftrightarrow x_{2}^{T} H A_{2} x_{2}=0 .
$$

The expressions $x_{1}^{T} H A_{1} x_{1}, x_{2}^{T} H A_{2} x_{2}$, viewed as functions of $H$, define linear functionals on the space of Hermitian matrices. Moreover, we have seen that the null sets of these functionals are identical. So they must be scalar multiples of each other. Furthermore, (15) implies that they are negative multiples of each other. That is,

$$
x_{1}^{T} H A_{1} x_{1}=-k x_{2}^{T} H A_{2} x_{2}
$$

with $k>0$, for all Hermitian matrices $H$.

It follows from elementary arguments [1 that either $x_{1}=\alpha x_{2}$ and $A_{1} x_{1}=$ $-\left(\frac{k}{\alpha}\right) A_{2} x_{2}$ or $x_{1}=\beta A_{2} x_{2}$ and $A_{1} x_{1}=-\left(\frac{k}{\beta}\right) x_{2}$. Consider the former situation to begin with. Then we have

$$
\begin{aligned}
A_{1}\left(\alpha x_{2}\right) & =-\left(\frac{k}{\alpha}\right) A_{2} x_{2} \\
\Rightarrow\left(A_{1}+\left(\frac{k}{\alpha^{2}}\right) A_{2}\right) x_{2} & =0
\end{aligned}
$$

and thus the pencil $\sigma_{\gamma[0, \infty)}\left[A_{1}, A_{2}\right]$ is singular. It follows that the matrix $A_{1} A_{2}^{-1}$ has a negative eigenvalue.

On the other hand, in the latter situation, we have that

$$
x_{2}=\frac{1}{\beta} A_{2}^{-1} x_{1}
$$

Thus

$$
\begin{aligned}
A_{1} x_{1} & =-\left(\frac{k}{\beta^{2}}\right) A_{2}^{-1} x_{1} \\
\Rightarrow\left(A_{1}+\left(\frac{k}{\beta^{2}}\right) A_{2}^{-1}\right) x_{1} & =0
\end{aligned}
$$

Thus, in this case the pencil $\sigma_{\gamma[0, \infty)}\left[A_{1}, A_{2}^{-1}\right]$ is singular. It follows that the matrix $A_{1} A_{2}$ has a negative eigenvalue. This completes the proof of Theorem 3.1. 


\section{Applications of Main Result}

In this section, we describe a number of applications of Theorem 1 and the techniques outlined in the last section. First of all, we shall present two direct applications of the Theorem to the problem of CQLF existence for pairs of exponentially stable LTI systems. The general problem of CQLF existence for families of LTI systems is recognised as an analytical problem of great difficulty. While it can be solved efficiently numerically using linear matrix inequalities [9, closed-form necessary and sufficient conditions for the existence of a CQLF are currently only known for a few special cases of system classes; in particular, for the case of pairs of second order LTI systems [10, 11], and for pairs of $n$ dimensional systems whose systems matrices differ by a rank 1 matrix [12]. We shall show below that both of these important system classes satisfy the seemingly abstract conditions specified by Theorem 1] Later in the section, we shall see that the same ideas that led to the result of Theorem 1 can be successfully applied to the related question of common diagonal Lyapunov function (CDLF) existence for pairs of exponentially stable positive LTI systems of arbitrary dimension. In fact, we shall present a compact algebraic condition that is necessary and sufficient for a generic pair of such systems to have a CDLF.

\section{(i) Second order systems}

We now illustrate the use of Theorem 1 for pairs of stable second order LTI systems.

Let $\Sigma_{A_{1}}$ and $\Sigma_{A_{2}}$ be stable LTI systems with $A_{1}, A_{2} \in \mathbb{R}^{2 \times 2}$. The following facts follow trivially for second order systems.

(a) If a strong CQLF exists for $\Sigma_{A_{1}}$ and $\Sigma_{A_{2}}$ then the pencils $\sigma_{\gamma[0, \infty)}\left[A_{1}, A_{2}\right]$ and $\sigma_{\gamma[0, \infty)}\left[A_{1}, A_{2}^{-1}\right]$ are necessarily Hurwitz.

(b) If $A_{1}$ and $A_{2}$ satisfy the non-strict Lyapunov equations (5), (6) then the matrices $Q_{1}$ and $Q_{2}$ are both rank 1 (rank $n-1$ ).

(c) If a strong CQLF does not exist for $\Sigma_{A_{1}}$ and $\Sigma_{A_{2}}$ then a positive constant $d$ exists such that a strong CQLF exists for $\Sigma_{A_{1}-d I}$ and $\Sigma_{A_{2}}$. By continuity a non-negative $d_{1}<d$ exists such that $A_{1}-d_{1} I$ and $A_{2}$ satisfy Theorem 1 and one of the pencils $\sigma_{\gamma[0, \infty)}\left[A_{1}-d_{1} I, A_{2}\right]$ and $\sigma_{\gamma[0, \infty)}\left[A_{1}-d_{1} I, A_{2}^{-1}\right]$ is necessarily singular. Hence, it follows that one of the pencils $\sigma_{\gamma[0, \infty)}\left[A_{1}, A_{2}\right]$ and $\sigma_{\gamma[0, \infty)}\left[A_{1}, A_{2}^{-1}\right]$ is not Hurwitz.

Items (a)-(c) establish the following facts. Given two stable second order LTI systems $\Sigma_{A_{1}}$ and $\Sigma_{A_{2}}$, a necessary condition for the existence of a strong CQLF is that the pencils $\sigma_{\gamma[0, \infty)}\left[A_{1}, A_{2}\right]$ and $\sigma_{\gamma[0, \infty)}\left[A_{1}, A_{2}^{-1}\right]$ are Hurwitz. Conversely, a necessary condition for the non-existence of a strong CQLF is that one of the pencils $\sigma_{\gamma[0, \infty)}\left[A_{1}, A_{2}\right]$ and $\sigma_{\gamma[0, \infty)}\left[A_{1}, A_{2}^{-1}\right]$ is not Hurwitz. Together these conditions yield the following known result [10]:

$A$ necessary and sufficient condition for the LTI systems $\Sigma_{A_{1}}$ and $\Sigma_{A_{2}}$, $A_{1}, A_{2} \in \mathbb{R}^{2 \times 2}$, to have a strong $C Q L F$ is that the pencils $\sigma_{\gamma[0, \infty)}\left[A_{1}, A_{2}\right]$ and $\sigma_{\gamma[0, \infty)}\left[A_{1}, A_{2}^{-1}\right]$ are Hurwitz. 
(ii) The SISO Circle Criterion

By modifying the argument presented in item (i) above a time domain formulation of the Circle Criterion can be obtained using Theorem 1

Let $\Sigma_{A_{1}}$ and $\Sigma_{A_{2}}$ be stable LTI systems with $A_{1}, A_{2} \in \mathbb{R}^{n \times n}$ and $\operatorname{rank}\left(A_{1}\right.$ $\left.A_{2}\right)=1$. Then, the following facts follow directly [3].

(a) If a strong CQLF exists for $\Sigma_{A_{1}}$ and $\Sigma_{A_{2}}$ then $\sigma_{\gamma[0, \infty)}\left[A_{1}, A_{2}^{-1}\right]$ and $\sigma_{\gamma[0, \infty)}\left[A_{1}, A_{2}\right]$ are necessarily Hurwitz.

(b) If $A_{1}$ and $A_{2}$ satisfy the non-strict Lyapunov equations (5) (6) then it is shown in 3] that the matrices $Q_{1}$ and $Q_{2}$ are both generically rank 1 (rank $n-1)$.

(c) If a strong CQLF does not exist for $\Sigma_{A_{1}}$ and $\Sigma_{A_{2}}$ then a positive constant $k$ exists such that a strong CQLF exists for $\Sigma_{A_{1}}$ and $\Sigma_{A_{2}+k\left(A_{1}-A_{2}\right)}$. By continuity a non-negative $k_{1}<k$ exists such that $A_{1}$ and $A_{2}+k_{1}\left(A_{1}-A_{2}\right)$ satisfy Theorem 1 and the pencil $\sigma_{\gamma[0, \infty)}\left[A_{1}^{-1}, A_{2}+k_{1}\left(A_{1}-A_{2}\right)\right]$ is necessarily singular.

(d) Let $A_{1}, B \in \mathbb{R}^{n \times n}$ with $A_{1}$ Hurwitz and $\operatorname{rank}(B)=1$. Suppose that for some $\lambda_{0}>0$, the matrix product $A_{1}\left(A_{1}+\lambda_{0} B\right)$ has a negative eigenvalue (the pencil $\sigma_{\gamma[0, \infty)}\left[A_{1}^{-1}, A_{1}+\lambda_{0} B\right]$ is singular). Then for all $\lambda>\lambda_{0}$, the product $A_{1}\left(A_{1}+\lambda B\right)$ has a negative eigenvalue (the pencil $\sigma_{\gamma[0, \infty)}\left[A_{1}^{-1}, A_{1}+\lambda B\right]$ is singular).

Items (a)-(d) establish the following result. Given two stable LTI systems $\Sigma_{A_{1}}$ and $\Sigma_{A_{2}}$ with $\operatorname{rank}\left(A_{1}-A_{2}\right)=1$, a necessary and sufficient condition for the existence of a strong CQLF is that the pencil $\sigma_{\gamma[0, \infty)}\left[A_{1}^{-1}, A_{2}\right]$ is non-singular. More formally:

Theorem 2. Let $A, A+B$ be two Hurwitz matrices in $\mathbb{R}^{n \times n}$ where $\operatorname{rank}(B)=1$. Then a necessary and sufficient condition for a strong CQLF to exist for the systems $\Sigma_{A}, \Sigma_{A+B}$ is that the matrix product $A(A+B)$ has no negative eigenvalues or equivalently, that the matrix pencil $\sigma_{\gamma[0, \infty)}\left[A^{-1}, A+B\right]$ is non-singular.

(iii) CDLF existence for positive linear systems

Recently, in [13 the same techniques that have been used above to derive Theorem 1 and to obtain the conditions for CQLF existence for pairs of LTI systems with system matrices differing by rank one and second order systems, have been applied to the problem of common diagonal Lyapunov function (CDLF) existence for pairs of so-called positive LTI systems. The class of positive systems, whose state variables are constrained to be non-negative for all time, is recognised to be of considerable practical importance, and examples of positive systems commonly occur in areas such as population dynamics, communication systems, pharmaceutics and economics [14. While, the theory of positive LTI systems is now well-developed [14, recent applications in areas such as congestion control of the Internet and formation flying [15, 16] have indicated the need for a greater understanding of time-varying, and in particular switched positive linear systems. As with general switched linear systems, several fundamental questions relating to the stability of positive switched linear systems are currently unresolved. 
Before stating the result on CDLF existence, we need to briefly provide some background on the theory of positive LTI systems. Firstly, it is well-known that an LTI system $\Sigma_{A}$ is positive if and only if the system matrix $A$ is a so-called Metzler matrix, where a Metlzer matrix $A$ in $\mathbb{R}^{n \times n}$ is one all of whose offdiagonal entries are non-negative, $a_{i j} \geq 0$ for $i \neq j$. A remarkable property of such systems is that a positive LTI system is exponentially stable if and only if it has a diagonal Lyapunov function. Thus, for any Metzler, Hurwitz matrix $A$ in $\mathbb{R}^{n \times n}$, there is some positive definite diagonal matrix $D$ such that $A^{T} D+D A<0$. This fact gives rise to the problem of determining when a CDLF exists for two or more exponentially stable positive LTI systems. Formally, given the family of exponentially stable positive LTI systems $\Sigma_{A_{1}}, \ldots, \Sigma_{A_{k}}$, where $A_{i} \in \mathbb{R}^{n \times n}, 1 \leq i \leq k$, if there exists some positive definite diagonal matrix $D$ such that

$$
A_{i}^{T} D+D A_{i}<0 \quad \text { for } 1 \leq i \leq k,
$$

then $V(x)=x^{T} D x$ is a CDLF for the systems $\Sigma_{A_{1}}, \ldots, \Sigma_{A_{k}}$. We shall now consider this problem for a pair of exponentially stable positive LTI systems under the mild assumption that their system matrices are irreducible [17 18.

Now let $\Sigma_{A_{1}}, \Sigma_{A_{2}}$ be exponentially stable positive LTI systems, where $A_{1}, A_{2}$ are irreducible, Metzler, Hurwitz matrices in $\mathbb{R}^{n \times n}$. Then the following points were established in 13 .

(a) If $\Sigma_{A_{1}}, \Sigma_{A_{2}}$ have a CDLF, then so do $\Sigma_{A_{1}}, \Sigma_{D A_{2} D}$ for all positive diagonal $D$ in $\mathbb{R}^{n \times n}$. Thus, $A_{1}+D A_{2} D$ must be Hurwitz and hence non-singular for all diagonal $D>0$.

(b) If there is no CDLF for $\Sigma_{A_{1}}, \Sigma_{A_{2}}$ but there exists a non-zero diagonal $D \geq 0$ satisfying

$$
A_{i}^{T} D+D A_{i}=Q_{i} \leq 0 \quad i \in\{1,2\}
$$

then $Q_{1}$ and $Q_{2}$ must have rank $n-1$. This is the crucial stage in the derivation of the condition for CDLF existence given in [13] and is interesting to note in the light of the hypotheses of Theorem 1 above.

(c) Following arguments analogous to those used in the derivation of Theorem 1 it is possible to show that in the situation described in (b) there is some diagonal $D>0$ such that $A_{1}+D A_{2} D$ is singular.

(d) Finally, if $\Sigma_{A_{1}}, \Sigma_{A_{2}}$ have no CDLF, then for $\alpha>0$ sufficiently large, $\Sigma_{A_{1}-\alpha I}$, $\Sigma_{A_{2}}$ will have a CDLF. If we then define

$$
\alpha_{0}=\inf \left\{\alpha>0: \Sigma_{A_{1}-\alpha I}, \Sigma_{A_{2}} \text { have a CDLF }\right\} \text {, }
$$

then $\Sigma_{A_{1}-\alpha_{0} I}, \Sigma_{A_{2}}$ satisfy the conditions of (b). It follows that there is some diagonal $D>0$ such that $A_{1}-\alpha_{0} I+D A_{2} D$ is singular. A suitable rescaling $\bar{D}$ of this $D$ will now make $A_{1}+D A_{2} D$ singular.

Taking points (a)-(d) together, we have the following result giving a necessary and sufficient condition for the existence of a CDLF for a generic pair of exponentially stable positive LTI systems. 
Theorem 3. [13] Let $\Sigma_{A_{1}}, \Sigma_{A_{2}}$ be exponentially stable positive LTI systems, where $A_{1}, A_{2}$ are irreducible Hurwitz, Metzler matrices in $\mathbb{R}^{n \times n}$. Then a necessary and sufficient condition for $\Sigma_{A_{1}}, \Sigma_{A_{2}}$ to have a CDLF is that $A_{1}+D A_{2} D$ is non-singular for all diagonal $D>0$.

\section{Implications of Main Result for General Multiplier Criteria}

Theorem 3.2 provides a time-domain condition for the existence of a CQLF for pairs of LTI systems whose system matrices differ by a rank 1 matrix. Alternative, but equivalent, conditions can be obtained using the SISO circle criterion. This observation raises the question as to whether time domain conditions derived using Theorem 2.1 can be obtained directly from classical frequency domain results. The following theorem provides an affirmative answer to this question.

Theorem 4. [2, 4] Let $G(j \omega)=\frac{N(j \omega)}{D(j \omega)}$ be a rational transfer function and $K \in \mathbb{R}^{+}$. Let $\{A, b, c, d\}$ be a controllable realisation of $G(j \omega)$ so that $G(j \omega)=$ $c^{T}(j \omega I-A)^{-1} b+d$. Let $A$ and $A-\frac{b c^{T}}{K+d}$ be strictly Hurwitz. Then, a necessary and sufficient condition for

$$
K+\operatorname{Re}\{G(j \omega)\}>0, \forall \omega \in \mathbb{R} \cup\{\infty\},
$$

is that the matrix-product $A\left(A-\frac{b c^{T}}{K+d}\right)$ has no negative real eigenvalues.

Outline of proof: Equation (17) can be written

$$
K+d+\operatorname{Re}\left\{c^{T}\left(j \omega I_{n}-A\right)^{-1} b\right\}>0
$$

for all $\omega \in \mathbb{R} \cup\{\infty\}$. In particular $K+d>0$. Applying Lemma 2 we obtain

$$
\begin{aligned}
K+d-1+\frac{\left(1-c^{T} A\left(\omega^{2} I_{n}+A^{2}\right)^{-1} b\right) \operatorname{det}\left[\omega^{2} I_{n}+A^{2}\right]}{\operatorname{det}\left[\omega^{2} I_{n}+A^{2}\right]} & >0 \\
K+d-c^{T} A\left(\omega^{2} I_{n}+A^{2}\right)^{-1} b & >0
\end{aligned}
$$

which implies

$$
\operatorname{det}\left[K+d-c^{T} A\left(\omega^{2} I_{n}+A^{2}\right)^{-1} b\right]>0 .
$$

Applying Lemma 1 yields

$$
\begin{aligned}
\operatorname{det}\left[(K+d) I_{n}-\left(\omega^{2} I_{n}+A^{2}\right)^{-1} b c^{T} A\right] & >0 \\
\operatorname{det}\left[\left(\omega^{2} I_{n}+A^{2}\right)^{-1}\right] \operatorname{det}\left[(K+d)\left(\omega^{2} I_{n}+A^{2}\right)-b c^{T} A\right] & >0 \\
\operatorname{det}\left[\left(\omega^{2} I_{n}+A^{2}\right)^{-1}\right](K+d) \operatorname{det}\left[\omega^{2} I_{n}+A^{2}-\frac{1}{K+d} b c^{T} A\right] & >0 \\
\operatorname{det}\left[\omega^{2} I_{n}+\left(A-\frac{1}{K+d} b c^{T}\right) A\right] & >0
\end{aligned}
$$


with the latter following as $K+d>0$ and $A$ has no imaginary eigenvalues. It follows that a necessary condition for (17) is that the product $A\left(A-\frac{1}{K+d} b c^{T}\right)$ has no negative eigenvalues.

Let $A_{1}=A$ and $A_{2}=A-\frac{1}{K+d} b c^{T}$ and suppose that $A_{2} A_{1}$ has no negative real eigenvalue, and that $A_{1}$ and $A_{2}$ are Hurwitz. It follows using the above argument in reverse that (17) holds.

Theorem 4 has profound implications for a number of classical frequency domain stability results derived in the context of the Lur'e problem. Lur'e considered the problem of determining the global asymptotic stability of the equilibrium state of the system:

$$
\begin{aligned}
\Gamma: \dot{x} & =A x+b u \\
u & =-f(\sigma, t) \\
\sigma & =c^{T} x
\end{aligned}
$$

where $A \in \mathbb{R}^{n \times n}$ is a Hurwitz matrix, $b, c \in \mathbb{R}^{n \times 1}$ and where $k_{1} \sigma^{2} \leq f(\sigma, t) \sigma \leq$ $k_{2} \sigma^{2}$. In the context of this problem three of the best known results in systems theory were derived; namely; the Kalman-Yacubovich-Popov lemma; the Circle Criterion; and the Popov Criterion. All three of these results establish conditions for stability as a constraint on the rational transfer function $G(j \omega)=c^{T}(j \omega-$ $A)^{-1} b$.

(i) The Kalman-Yacubovich-Popov (KYP) Lemma : The single-input singleoutput (SISO) version of the Kalman-Yacubovich-Popov lemma [7] is expressed in the form of a strictly positive real (SPR) condition: namely,

$$
\gamma+\operatorname{Re}\left\{c^{T}\left(j \omega I_{n}-A\right)^{-1} b\right\}>0 \forall \omega \in \mathbb{R},
$$

for some $\gamma \in \mathbb{R}^{+}$. A necessary condition for the above inequality to hold is that both $A$ and $A-\frac{1}{\gamma} b c^{T}$ are Hurwitz. Hence, given this fact, it follows from Theorem 4 that a time-domain version of the SPR condition for SISO systems is that the matrix $A\left(A-\frac{1}{\gamma} b c^{T}\right)$ does not have any negative real eigenvalues.

(ii) The Circle Criterion [2]: The SISO version of the circle criterion is derived directly from the SISO KYP lemma. Here, conditions are derived for the existence of a Lyapunov function $V(x)=x^{T} P x, P=P^{T} \in \mathbb{R}^{n \times n}$ for the non-linear Lur'e type system $\Gamma$. In the case where $k_{1}=0$ and $k_{2}=1 \mathrm{a}$ necessary and sufficient condition for the existence of a quadratic Lyapunov function $V(x)$ is that [12]

$$
1+\operatorname{Re}\left\{c^{T}\left(j \omega I_{n}-A\right)^{-1} b\right\}>0 \forall \omega \in \mathbb{R} .
$$

It follows from Theorem 4 that a time-domain version of the circle criterion with $0 \leq f(\sigma, t) \leq 1$ is that the matrix $A\left(A-b c^{T}\right)$ does not have any negative real eigenvalues ( $A$ and $A-b c^{T}$ are necessarily Hurwitz as before).

(iii) The Popov Criterion: The SISO Popov criterion [19] considers the stability of the system $\Gamma$ where the nonlinearity $f$ is time-invariant. A sufficient 
condition for the absolute stability of this system is that there exists a strictly positive $\alpha \in \mathbb{R}$ such that

$$
\frac{1}{k}+\operatorname{Re}\left\{(1+j \alpha \omega) c^{T}\left(j \omega I_{n}-A\right)^{-1} b\right\}>0 \forall \omega \in \mathbb{R} .
$$

It follows from Theorem 4 that a time-domain version of the Popov criterion can be stated as follows: there exists a positive $\alpha \in \mathbb{R}$ such that the matrix $\bar{A}\left(\bar{A}-\frac{1}{d+\frac{1}{k}} \bar{b} \bar{c}\right)$ does not have any negative real eigenvalues where $\{\bar{A}, \bar{b}, \bar{c}, \bar{d}\}$ is the control canonical form of $(1+j \alpha \omega) c^{T}\left(j \omega I_{n}-A\right)^{-1} b$ and that both these matrices are Hurwitz.

Comment: The Popov criterion is an example of a multiplier criterion. Over the past 40 years many authors, including Popov, Zames and Falb, Willems, Narendra and Taylor, and many others [20] 21, 22] have developed stability multiplier criteria that exploit additional assumed properties of the sector nonlinearity $f$. Roughly speaking, these conditions are expressed in the form of a strictly positive real condition on a function of the form of

$$
\gamma+\operatorname{Re}\left\{c^{T}\left(j \omega I_{n}-A\right)^{-1} b\right\}>0 \forall \omega \in \mathbb{R} .
$$

Consequently, compact time-domain versions of these criteria can be obtained using Theorem 4. A particularly useful consequence of our result is that it leads to very compact conditions for checking strict positive realness of a given LTI system [23].

\section{$6 \quad$ Examples}

Example 1. Consider the non-linear system

$$
\begin{aligned}
\Gamma: \dot{x} & =A x+b u \\
u & =-f(\sigma) \\
\sigma & =c^{T} x
\end{aligned}
$$

where

$$
A=\left[\begin{array}{cc}
0 & 1 \\
-1 & -2
\end{array}\right], b=\left[\begin{array}{l}
0 \\
1
\end{array}\right], c=\left[\begin{array}{l}
1 \\
0
\end{array}\right],
$$

and where $0 \leq f(\sigma) \sigma \leq \sigma^{2}$. This system may be analysed using both the Circle and Popov Criterion.

(i) The Circle Criterion : The matrix product $W=A\left(A-b c^{T}\right)$ is given by

$$
W=\left[\begin{array}{cc}
-2 & -2 \\
4 & 3
\end{array}\right]
$$

Since $W$ does not have any negative eigenvalues it follows from the Circle Criterion that the system $\Gamma$ is absolutely stable. 
(ii) The Popov Criterion: The control canonical form of $(1+j \alpha \omega) c^{T}\left(j \omega I_{n}-\right.$ $A)^{-1} b$ is:

$$
\bar{A}=\left[\begin{array}{cc}
0 & 1 \\
-1 & -2
\end{array}\right], \bar{b}=\left[\begin{array}{l}
0 \\
1
\end{array}\right], \bar{c}=\left[\begin{array}{l}
1 \\
\alpha
\end{array}\right], \bar{d}=0 .
$$

The matrix product $W(\alpha)=\bar{A}\left(\bar{A}-\bar{b} \bar{c}^{T}\right)$ is given by

$$
W(\alpha)=\left[\begin{array}{cc}
-2 & -2-\alpha \\
4 & 3+2 \alpha
\end{array}\right] .
$$

The matrix $W(1)$ does not have negative real eigenvalues. Hence, it also follows from the Popov Criterion that the system $\Gamma$ is absolutely stable.

Example 2. Consider the stable dynamic systems $\Sigma_{A_{1}}$ and $\Sigma_{A_{2}}$ with:

$$
A_{1}=\left[\begin{array}{ccc}
0 & 1 & 0 \\
0 & 0 & 1 \\
-1 & -2 & -3
\end{array}\right], \quad A_{2}=\left[\begin{array}{ccc}
0 & 1 & 0 \\
0 & 0 & 1 \\
-2 & -3 & -1
\end{array}\right] \text {. }
$$

The matrix product $A_{1} A_{2}$ is given by:

$$
A_{1} A_{2}=\left[\begin{array}{ccc}
0 & 0 & 1 \\
-2 & -3 & -1 \\
6 & 8 & 1
\end{array}\right] \text {. }
$$

A CQLF cannot exist for $\Sigma_{A_{1}}$ and $\Sigma_{A_{2}}$ as the eigenvalues of $A_{1} A_{2}$ are given by $\lambda_{i}=\{1,-2,-1\}$.

\section{Open Questions}

In this section, we briefly discuss two major open questions that arise out of the work described earlier in the paper, and that should form the subject of future research.

Identification of system classes that satisfy Theorem [1:

We have seen above how Theorem 1 unifies, in a certain sense, the results on CQLF existence previously derived for second order systems and systems whose system matrices differ by rank one. In both of these cases, the conditions for CQLF existence are easy to verify and can be interpreted in terms of the dynamics of the associated switched linear systems. Moreover, the form of the conditions given in the conclusions of Theorem 1 suggest that for any class of system to which the Theorem can be applied, it may be possible to derive similarly attractive conditions for CQLF existence using analogous techniques to those employed above. Given the need for verifiable conditions that can be used to determine the stability of switched linear systems, this observation leads to the important problem of identifying further classes of systems to which Theorem 廿 can be applied. The discovery of such system classes is likely to lead to other 
results along the lines of Theorem 2 giving verifiable, dynamically meaningful conditions for CQLF existence.

Extension of convex set techniques to non-quadratic Lyapunov functions:

In general, the existence of a strong CQLF is only a sufficient condition for the exponential stability of switched linear systems under arbitrary switching, and in certain situations, less conservative results may be obtained based on nonquadratic Lyapunov functions. Much recent work in this direction has focussed on Lyapunov functions, defined using vector norms, that are piecewise linear or piecewise quadratic. For the analysis of switching systems, the fact that these functions can be non-smooth is an advantage, as the switching action itself is non-smooth in nature.

The $l_{1}$-norm based Lyapunov function

$$
V(x)=\|W x\|_{1} \quad W \in \mathbb{R}^{2 \times 2}
$$

was proposed in [24]. Such functions are referred to as unic Lyapunov functions. Note that while the LTI system $\Sigma_{A}$ has a quadratic Lyapunov function if and only if the eigenvalues of $A$ lie in the open left half plane, a unic Lyapunov function exists for the system if and only if the eigenvalues of $A$ lie within the so-called $45^{\circ}$ region

$$
\{z \in \mathbb{C}:|\operatorname{Im}(z)|<|\operatorname{Re}(z)|, \operatorname{Re}(z)<0\} .
$$

In 25], conditions for common unic Lyapunov function existence for pairs of second order LTI systems are described. These conditions are related to the matrix-pencil conditions given for CQLF existence for the second-order case in Section 4 above.

The conditions for common unic Lyapunov function existence for second order systems were derived using direct algebraic arguments specific to the second order case. In the light of the work described earlier on the CQLF existence problem, a natural question to ask is whether similar convex-cone based techniques can be applied in this setting to obtain more general results on common unic Lyapunov function existence. In order to do this, we would need to obtain a greater understanding of the set of unic Lyapunov functions corresponding to a given stable LTI system, and then investigate the possibility of obtaining results similar to Theorem 1 for unic Lyapunov functions.

\section{Concluding Remarks}

In this paper we have presented a result on common quadratic Lyapunov functions. We have shown that this result unifies a number of classical stability results and leads to time-domain versions of a number of known frequency-domain stability criteria. As well as addressing the two open problems described in the paper, future research will proceed in a number of directions; (i) by classifying the classes of systems that satisfy the assumptions of Theorem 1 (ii) by exploring the potential of time-domain stability criteria in for deriving stable adaptive 
control systems; and (iii) by using the time-domain stability criteria to reinterpret classical frequency domain analysis. Work in all three directions is ongoing and will be reported in future publications.

Acknowledgements This paper first appeared in part at the 12th Yale Workshop on Adaptive and Learning systems [26]. The authors gratefully thank Professor K. S. Narendra for permission to reproduce parts of this paper. This work was partially supported by the European Union funded research training network Multi-Agent Control, HPRN-CT-1999-001072, by the Enterprise Ireland grant SC/2000/084/Y, and by Science Foundation Ireland under the grant 00/PI.1/C067. Neither the European Union or Enterprise Ireland is responsible for any use of data appearing in this publication.

\section{References}

[1] R. Shorten, K. Narendra, and O. Mason, "A result on common Lyapunov functions," IEEE Transactions on Automatic Control, vol. 48, no. 1, pp. 110-113, 2003.

[2] R. Shorten and K. Narendra, "On common quadratic Lyapunov functions for pairs of LTI systems whose system matrices are in companion form," IEEE Transactions on Automatic Control, vol. 48, no. 4, pp. 618-622, 2003.

[3] R. Shorten, O. Mason, F. O'Cairbre, and P. Curran, "A unifying result for the circle criterion and other stability criteria." Accepted for publication in proceedings of the European Control Conference (extended version submitted to International Journal of Control), 2003.

[4] R. Shorten, P. Curran, and K. Wulff, "On time domain multiplier criteria for SISO systems." Submitted to Automatica, 2003.

[5] R. Loewy, "On ranges of real Lyapunov transformations," Linear Algebra and its Applications, vol. 13, no. 1, pp. 79-89, 1976.

[6] G. P. Barker, A. Berman, and R. J. Plemmons, "Positive Diagonal Solutions to the Lyapunov Equations," Linear and Multilinear Algebra, vol. 5, no. 3, pp. 249-256, 1978.

[7] R. E. Kalman, "Lyapunov functions for the problem of Lur'e in automatic control," Proceedings of the national academy of sciences, vol. 49, no. 2, pp. 201-205, 1963.

[8] T. Kailath, Linear Systems. Prentice Hall, New Jersey, 1980.

[9] S. Boyd and Q. Yang, "Structured and simultaneous Lyapunov functions for system stability problems," International Journal of Control, vol. 49, no. 6, pp. 2215$2240,1989$.

[10] R. Shorten and K. S. Narendra, "Necessary and sufficient conditions for a CQLF for a finite number of stable second order LTI systems," International Journal of Adaptive Control and Signal Processing, vol. 16, no. 9, pp. 709-728, 2003.

[11] N. Cohen and I. Lewkowicz, "A necessary and sufficient criterion for the stability of a convex set of matrices," IEEE Transactions on Automatic Control, vol. 38, no. 4, pp. 611-615, 1993.

${ }^{2}$ This work is the sole responsibility of the authors and does not reflect the European Union's opinion 
[12] K. S. Narendra and R. M. Goldwyn, "A geometrical criterion for the stability of certain non-linear non-autonomous systems," IEEE Transactions on Circuit Theory, vol. 11, no. 3, pp. 406-407, 1964.

[13] O. Mason and R. Shorten, "On the simultaneous diagonal stability of a pair of positive linear systems," submitted to Linear Algebra and its Applications, 2004.

[14] L. Farina and S. Rinaldi, Positive linear systems. Wiley Interscience Series, 2000.

[15] R. Shorten, D. Leith, J. Foy, and R. Kilduff, "Towards an analysis and design framework for congestion control in communication networks," in Proceedings of the 12th Yale workshop on adaptive and learning systems, 2003.

[16] A. Jadbabaie, J. Lin, and A. S. Morse, "Co-ordination of groups of mobile autonomous agents using nearest neighbour rules," IEEE Transactions on Automatic Control, vol. 48, no. 6, pp. 988-1001, 2003.

[17] A. Berman and R. Plemmon, Non-negative matrices in the mathematical sciences. SIAM classics in applied mathematics, 1994.

[18] R. Horn and C. Johnson, Matrix Analysis. Cambridge University Press, 1985.

[19] J. Slotine and W. Li, Applied Nonlinear Control. Prentice Hall, 1991.

[20] K. Narendra and J. Taylor, Frequency Domain Criteria for Absolute Stability. Academic Press, 1973.

[21] G. Zames and P. L. Falb, "Stability conditions for systems with monotone and slope restricted non-linearities," SIAM Journal of Control and Optimization, vol. 6 , pp. 89-108, 1968.

[22] J. L. Willems, "The circle criterion and quadratic Lyapunov functions for stability analysis," IEEE Transactions on Automatic Control, vol. 18, no. 4, p. 184, 1973.

[23] R. Shorten and C. King, "Spectral conditions for strict positive realness of lti systems." Accepted for publication in IEEE Transactions on Automatic Control, 2004.

[24] K. Wulff, R. N. Shorten, and P. Curran, "On the relationship of matrix-pencil eigenvalue criteria and the choice of Lyapunov function for the analysis of second order switched systems," in American Control Conference, 2002.

[25] K. Wulff, R. Shorten, and P. Curran, "On the 45 degree region and the uniform asymptotic stability of classes of second order parameter varying and switched systems," International Journal of Control, vol. 75, no. 11, pp. 812-823, 2002.

[26] R. Shorten, K. S. Narendra, O. Mason, and K. Wulff, "On the existence of a common quadratic Lyapunov functions for SISO swithed systems," in proceedings of Tweltfth Yale Workshop on Adaptive and Learning Systems, 2003. 